\title{
Outpatient parenteral antimicrobial therapy with ceftriaxone for acute tonsillopharyngitis: efficacy, patient satisfaction, cost effectiveness, and safety
}

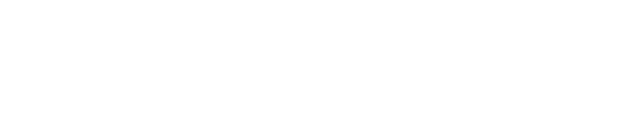

\author{
Samah Al Alawi' \\ Somaya Abdulkarim' \\ Hazem Elhennawy' \\ Anwar Al-Mansoor ${ }^{2}$ \\ Ahmed Al Ansari ${ }^{3,4,5}$ \\ 'Department of Family Medicine, \\ ${ }^{2}$ Department of Dietetics and \\ Nutrition, ${ }^{3}$ Training and Education \\ Department, Bahrain Defence \\ Force Hospital, Riffa, ${ }^{4}$ Arabian Gulf \\ University, Manama, ${ }^{5}$ Royal College \\ of Surgeons of Ireland, Busaiteen, \\ Kingdom of Bahrain
}

Background: Outpatient parenteral antimicrobial therapy (OPAT) is the administration of intravenous antimicrobial therapy to patients in an outpatient setting. It may be used for patients who have infections that require parenteral treatment but who are otherwise stable enough to not require admission as inpatients.

Objective: We aimed to review the treatment of patients with acute tonsillopharyngitis at the OPAT health care clinic in the Bahrain Defense Force Royal Medical Services (BDF-RMS), with regard to efficacy, patient satisfaction, cost effectiveness, and safety.

Methods: A retrospective case notes review was conducted for all patients admitted to the OPAT clinic in the BDF-RMS with acute tonsillopharyngitis treated with ceftriaxone, between March 2012 and March 2014.

Results: In the period between March 2012 and March 2014, 97 patients with acute tonsillopharyngitis were treated with ceftriaxone for a minimum of 3 days at the OPAT clinic. In total, $94.8 \%$ of patients completed the prescribed course of ceftriaxone. Total cure was achieved in $89.7 \%$ of patients. Usage of the OPAT clinic led to cost savings of $10,693 \mathrm{BD}$, while total bed days saved were 301 over the 2-year period examined by this study. Participants in the program expressed high satisfaction rates, and the average ( \pm standard deviation) score on a patient satisfaction survey was $4.41( \pm 0.31)$ out of a total of 5 . This study highlights the efficacy, patient satisfaction, cost effectiveness, and safety of the OPAT clinic service for the treatment of acute tonsillopharyngitis with ceftriaxone. We found a $45.5 \%$ drop in admission rate for acute tonsillopharyngitis after starting the OPAT service clinic and that 301 bed days were saved through this treatment.

Conclusion: This study showed that the management of acute tonsillopharyngitis with ceftriaxone in the OPAT clinic is safe, clinically effective, and cost effective, with low rates of complications/readmissions and high levels of patient satisfaction.

Keywords: OPAT, ceftriaxone, tonsillopharyngitis

\section{Introduction}

Outpatient parenteral antimicrobial therapy (OPAT) was first established in practice in the United States in 1974 and has been growing rapidly into mainstream practice ever since. ${ }^{1}$ OPAT is the administration of intravenous (IV) antimicrobial therapy to patients in an outpatient setting. It may be used for patients who have infections that require parenteral treatment but who are otherwise stable enough to not require admission as inpatients. High levels of patient satisfaction have been documented with OPAT services, and they have also proven to be cost effective in most health care settings. ${ }^{2}$
Correspondence: Ahmed Al Ansari Training and Education Department, Bahrain Defense Force Hospital, Off Waly Alahed Avenue, PO Box 28743, West Riffa, Kingdom of Bahrain Tel +973 17766666

Email ahmed.alansari@bdfmedical.org 
Patients in the OPAT clinic are monitored clinically by a consultant family physician along with a well-trained nurse. The OPAT clinic service differs from inpatient care in that it provides the same care and treatment the patient would receive as an inpatient, without the need for an overnight hospital stay.

OPAT clinics are relatively new to the Middle East region. ${ }^{3}$ Our OPAT services clinic was established in the Bahrain Defense Force Royal Medical Services (BDFRMS) in the Kingdom of Bahrain in February 2012, with the overall goals of reducing patient hospital stays, lowering admission costs, and increasing patient satisfaction rates. ${ }^{3}$ The BDF-RMS hospital is a military teaching hospital that consists of both inpatient and outpatient services, with eight beds designated for the Ear, Nose, and Throat (ENT) department.

The OPAT clinic of the BDF-RMS is the first of its kind in Bahrain. It consists of five beds and is located in the outpatient general practice clinic, near the emergency department. OPAT patients are referred from both inpatient and outpatient hospital services, and acute tonsillopharyngitis is the most commonly referred diagnosis, accounting for $50 \%$ of OPAT patients.

A retrospective study conducted in Hinchingbrooke found that ceftriaxone was the most frequently prescribed drug in OPAT clinics, a practice that was similar to that used in our clinic, as our most commonly prescribed antibiotic in the OPAT clinic was also found to be third-generation cephalosporin, Ceftriaxone. ${ }^{4}$

Acute tonsillopharyngitis with viral infection is often symptomatically treated. When there is a secondary bacterial infection with accumulation of pus on the tonsils, penicillin is often used as the primary drug of choice by many physicians, although a recent study has found amoxicillin is less effective as a treatment for this condition relative to other drugs. Namely, cephalosporin, either alone or in combination with metronidazole, has been found to be a superior drug to penicillin, with high clinical efficacy in treating acute tonsillopharyngitis. ${ }^{5}$

A short-term therapy study found that approximately $100 \%$ of patients with streptococcal tonsillopharyngitis treated with ceftriaxone were clinically cured, and 95\% of these patients had pharyngeal sterilization. ${ }^{6}$ The failure of oral penicillin combined with second-generation cephalosporin to treat acute tonsillopharyngitis suggests that the strains present in our region may have developed resistance to these particular drugs. As such, a simpler and shorter OPAT regimen using third-generation cephalosporin (ceftriaxone) for 3-5 days may be more effective as an alternative to admission to the ENT ward.

Therefore, the aim of this study was to determine the efficacy, patient satisfaction, cost effectiveness, and safety of patients with acute tonsillopharyngitis who received ceftriaxone in the BDF-RMS OPAT clinic.

\section{Methods}

A retrospective analysis of previously collected data regarding OPAT patients with acute tonsillopharyngitis treated with ceftriaxone between March 2012 and March 2014 was completed at the BDF-RMS.

OPAT patients were admitted into the OPAT clinic following medical and nursing assessments according to a number of predefined criteria. Eligibility was established according to the ENT department guidelines for tonsillopharyngitis admission, which include: absolute dysphagia with moderate to severe dehydration; infections that are unresponsive to oral therapy; lack of improvement following 2 days of initial oral management; and finally, the presence of membranous tonsillitis. In addition, the eligibility criteria of the OPAT clinic require a patient be medically stable; have adequate venous access; be capable of understanding and consenting to OPAT; and have safe social circumstances with access to both telephone and transport. This study excluded patients who were under 2 years of age, as well as patients who were treated with drugs other than ceftriaxone.

Most of the patients received a full course of oral penicillin before they were started on IV ceftriaxone. Some of them had even received both penicillin and second-generation cephalosporin, such as Zinnat, without response. Throat cultures were not routinely recommended, and patients with a score of 4 or 5 according to the modified Centor criteria were treated empirically. The Centor criteria give an indication of the likelihood of a sore throat being due to bacterial infection.

Analytic parameters of the study included age, sex, nationality, dose, frequency, duration, route of administration, the indication for initiating ceftriaxone, oral antibiotic intake before initiation, relevant investigations (eg, complete blood count [CBC], throat swab), completion of the ceftriaxone course, and management outcome. Data were retrieved from an Excel $^{\circledR}$ spreadsheet specifically designed for this study, and patient case notes were reviewed to obtain complete data.

Patient safety issues were assured by following the OPAT guidelines for selecting candidates and through daily monitoring of patients by a multidisciplinary team, which included a family physician, an infectious disease consultant, and a 
specifically trained nurse. Patients were monitored for any adverse effects and complications during the period of antibiotic intake. The first dose was monitored in the OPAT clinic. In addition, written instructions were given to the patients or relatives to report to the accident and emergency department if any sign of adverse effects were noticed. Clinical efficacy was evaluated by examining the period of throat soreness, fever recovery, and number of return visits.

The total cost of treatment of outpatients with acute tonsillopharyngitis in the OPAT clinic was obtained from the billing department and compared with the total cost of the treatment of inpatients with the same condition receiving the same care.

Patient satisfaction was determined via a modified patient satisfaction questionnaire with 18 questions. The survey was modified and translated into Arabic; two-way translation was carried out to ensure consistent meaning.

The collected data were analyzed using SPSS version 19 (IBM Corp, Armonk, New York, NY, USA), and descriptive statistics were conducted. Reliability was examined using Cronbach's alpha.

\section{Ethics}

Approval was obtained from the Research and Research Ethics Committee at the BDF-RMS hospital.

\section{Results}

Acute tonsillopharyngitis is a common condition treated in our OPAT clinic. It has been found that tonsillopharyngitis is the main cause of approximately $15 \%$ of all visits to primary care physicians. ${ }^{7}$ Tonsillopharyngitis inpatient admissions at the BDF hospital were 972 within the 2-year period dating from March 1, 2010 to February 28, 2012 and reduced to approximately half that rate, to 530 , within the 2 years that directly followed the establishment of the OPAT clinic. This condition affects both children and adults, results in absenteeism from school and from work, and reduces the overall sense of quality of life for each patient affected. ${ }^{8}$

Over the 2-year period between March 2012 and February 2014, 97 patients with tonsillopharyngitis, 59 (60.8\%) males and $38(39.2 \%)$ females, were treated with ceftriaxone in the OPAT clinic. The highest number of patients was in the age group 15-45 years, and the lowest number was found in the age group 2-3 years. The sex and age distribution of patients with acute tonsillopharyngitis are shown in Figure 1 and Table 1.

The majority of patients were Bahraini (84 patients [86.6\%]), followed by Yemini (five patients [5.15\%]), Syrian

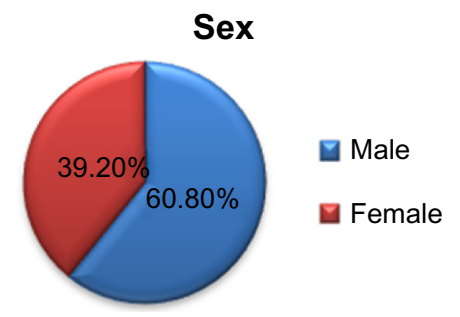

Figure I Sex distribution of OPAT patients treated with ceftriaxone. Note: In all, 59 (60.8\%) males and 38 (39.2\%) females were treated with ceftriaxone in the OPAT clinic.

Abbreviation: OPAT, outpatient parenteral antimicrobial therapy.

(three patients [3.1\%]), Indian (two patients [2.06\%]) Kuwaiti (one patient [1.03\%]), Egyptian (one patient [1.03\%]), and Norwegian (one patient [1.03\%]). The representation of Bahraini patients relative to non-Bahraini patients is presented in Figure 2, and the various nationalities of participants are shown in Table 2.

All patients with acute tonsillopharyngitis treated in the OPAT clinic included in this study received ceftriaxone, either through Intravenous (89 patients [91.8\%]) or intramuscular (eight patients $[8.2 \%]$ ) routes. The duration of ceftriaxone treatment was 3 days in 89 patients $(91.8 \%)$, while eight patients $(8.2 \%)$ received the treatment for more than 3 days. The dose of ceftriaxone was once or twice daily in 67 patients $(69.1 \%)$ and 30 patients $(30.9 \%)$, respectively. This data is represented in Table 3.

Parenteral ceftriaxone was initiated due to the following reasons: failure of oral therapy in 36 patients (37.1\%); inability to swallow in 24 patients $(24.7 \%)$; both inability to swallow and failure of oral antibiotic therapy in 21 patients (21.6\%); undocumented reason in 14 patients (14.4\%); and finally, referral from the private sector to continue previous treatment for two patients (2.1\%) (Table 4).

Although the failure of oral antibiotic therapy at 48 hours was one of the selection criteria, most of the patients had received a full course of antibiotics before starting the parenteral antibiotic. In addition, a small number started IV antibiotics after 2 days of oral antibiotics, due to clinical

Table I Age distribution of OPAT patients treated with ceftriaxone

\begin{tabular}{lll}
\hline Age group & Number of patients & \% of patients \\
\hline $2-3$ years & 4 & 4.1 \\
$4-14$ years & 15 & 15.5 \\
15-45 years & 73 & 75.3 \\
$>45$ years & 5 & 5.2 \\
Total & 97 & 100 \\
\hline
\end{tabular}

Notes: The highest number of patients was in the age group $15-45$ years, and the lowest number was found in the age group 2-3 years.

Abbreviation: OPAT, outpatient parenteral antimicrobial therapy. 


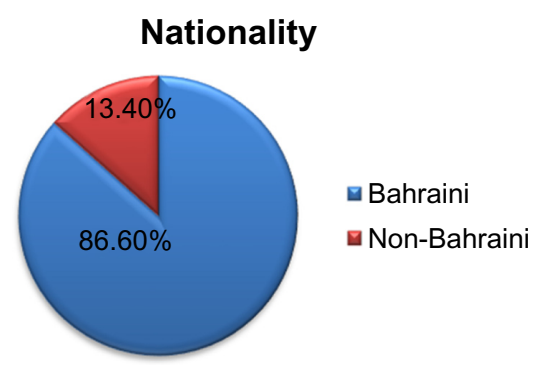

Figure 2 Nationality representation among the patients.

deterioration of the patient condition, increase in the neutrophils counts, and availability of culture results that showed resistance to penicillin.

In all, 54 patients received oral antibiotics before starting ceftriaxone. Out of the 54 patients who used oral antibiotics before initiating ceftriaxone, the most commonly failed oral antibiotic was Augmentin (28 patients), followed by combined antibiotics (nine patients), amoxicillin (seven patients), cefuroxime (five patients), and other antibiotics (five patients) (Table 5).

Nearly one-quarter (26.8\%) of patients did not use oral antibiotics before initiating treatment with ceftriaxone, while there was no information about prior treatment used in $17.5 \%$ of patients.

$\mathrm{CBC}$ results were collected from 77 patients $(79.4 \%)$. Thirty-eight patients presented with high white blood cell counts (defined as more than $12 \times 10^{9} / \mathrm{L}$ ), and 54 patients showed high neutrophils counts (defined as higher than $70 \%$ ). Throat swabs for culture and sensitivity were carried out in 32 patients (33\%). Of the 32 throat swabs collected, bacterial species were isolated in 11 cases. Group A betahemolytic Streptococcus was found in five cases, while the Staphylococcus sp. was also found in five other cases; both were the most commonly isolated organisms, followed by methicillin-resistant Staphylococcus aureus (MRSA) in one case (Table 6).

Table 2 Nationalities represented by patients

\begin{tabular}{lll}
\hline Nationalities & Number of patients & $\%$ of patients \\
\hline Bahraini & 84 & 86.6 \\
Yemeni & 5 & 5.15 \\
Syrian & 3 & 3.1 \\
Indian & 2 & 2.06 \\
Kuwaiti & 1 & 1.03 \\
Egyptian & 1 & 1.03 \\
Norwegian & 1 & 1.03 \\
Total & 97 & 100 \\
\hline
\end{tabular}

Notes: It was found that the majority of patients were Bahraini (84 [86.6\%]), followed by Yemini (five [5.15\%]), Syrian (three [3.1\%]), Indian (two [2.06\%]), Kuwaiti (one [1.03\%]), Egyptian (one [1.03\%]), and Norwegian (one [1.03\%]).
Out of a total of 97 patients, 92 patients (94.8\%) completed the course of ceftriaxone, while four patients were lost to follow up by the OPAT clinic. One patient showed improvement after the first dose and decided, against medical advice, not to complete the course, leaving a total of five patients who did not complete the ceftriaxone course.

Total cure was achieved in 87 patients $(89.7 \%)$, while six patients $(6.2 \%)$ were admitted to the ENT ward and four (4.1\%) were lost to follow up by the OPAT clinic.

The total cost for the treatment of acute tonsillopharyngitis with ceftriaxone in the OPAT clinic over a 24-month period was approximately 7,129 BHD. However, had these same patients been admitted to a general ward as inpatients, the cost would have been $17,822 \mathrm{BHD}$, a difference of 10,693 BHD. Moreover, if those patients had been admitted to a private ward, costs would have increased up to 20,792 BHD. Thus, by utilizing the OPAT clinic service, the hospital saved 10,693 BHD over 2 years. The total bed days saved over the same period was 301 .

Patient satisfaction was assessed using the short-form questionnaire presented in Table 7. Patient satisfaction was achieved by the OPAT services in treatment of acute tonsillopharyngitis with ceftriaxone. Patient satisfaction was measured through a five-point rating questionnaire. We achieved a response rate of $96.9 \%$, and patients expressed high satisfaction rates, of an average ( \pm standard deviation) score of $4.41( \pm 0.31)$ out of 5 . The reliability of our questionnaire was measured as Cronbach's alpha $=0.81$.

\section{Discussion}

This study highlights the efficacy, patient satisfaction, cost effectiveness, and safety of the OPAT clinic service for the treatment of acute tonsillopharyngitis with ceftriaxone. Several studies have reported the efficacy, safety, and acceptability of OPAT for the treatment of a wide variety of infections. ${ }^{9}$ Acute tonsillopharyngitis is one of the most common ENT infections presenting in primary care settings, ${ }^{9}$ and some cases may require parenteral therapy for hospital admission. Due to the feasibility and availability of the OPAT clinic in the BDF-RMS, the use of OPAT services that follow ENT guidelines present a better alternative than hospital admission to treat infections, such as acute tonsillopharyngitis.

Ceftriaxone was selected for the management of acute tonsillopharyngitis due to its excellent safety profile, low frequency of adverse events, broad-spectrum antimicrobial activity, and long half-life, rendering it one of the best treatment options for use in an OPAT setting. ${ }^{10}$ 
Table 3 Dose, duration, and route of treatment

\begin{tabular}{|c|c|c|c|c|c|c|c|c|c|c|c|}
\hline \multicolumn{4}{|l|}{$\begin{array}{l}\text { Dose of } \\
\text { ceftriaxone }\end{array}$} & \multicolumn{4}{|l|}{$\begin{array}{l}\text { Duration of } \\
\text { ceftriaxone }\end{array}$} & \multicolumn{4}{|l|}{$\begin{array}{l}\text { Route of } \\
\text { ceftriaxone }\end{array}$} \\
\hline \multicolumn{2}{|l|}{ Once daily } & \multicolumn{2}{|l|}{ Twice daily } & \multicolumn{2}{|l|}{3 days } & \multicolumn{2}{|l|}{$>3$ days } & \multicolumn{2}{|l|}{ Intravenous } & \multicolumn{2}{|c|}{ Intramuscular } \\
\hline $\begin{array}{l}\text { Number of } \\
\text { patients }\end{array}$ & $\begin{array}{l}\% \text { of } \\
\text { patients }\end{array}$ & $\begin{array}{l}\text { Number of } \\
\text { patients }\end{array}$ & $\begin{array}{l}\% \text { of } \\
\text { patients }\end{array}$ & $\begin{array}{l}\text { Number of } \\
\text { patients }\end{array}$ & $\begin{array}{l}\% \text { of } \\
\text { patients }\end{array}$ & $\begin{array}{l}\text { Number of } \\
\text { patients }\end{array}$ & $\begin{array}{l}\% \text { of } \\
\text { patients }\end{array}$ & $\begin{array}{l}\text { Number of } \\
\text { patients }\end{array}$ & $\begin{array}{l}\% \text { of } \\
\text { patients }\end{array}$ & $\begin{array}{l}\text { Number of } \\
\text { patients }\end{array}$ & $\begin{array}{l}\% \text { of } \\
\text { patients }\end{array}$ \\
\hline 67 & 69.1 & 30 & 30.9 & 89 & 91.8 & 8 & 8.2 & 89 & 91.8 & 8 & 8.2 \\
\hline
\end{tabular}

Notes: All patients with acute tonsillopharyngitis treated in the OPAT clinic included in this study received ceftriaxone, either through intravenous or intramuscular routes. The duration of ceftriaxone treatment was 3 days in the majority of patients, with some receiving treatment for more than 3 days. The dose of ceftriaxone was once or twice daily.

Abbreviation: OPAT, outpatient parenteral antimicrobial therapy.

Most of our patients received a full course of oral penicillin before they were started on IV ceftriaxone. Some of them even received penicillin and a second-generation cephalosporin, such as Zinnat, without response. With deterioration of the clinical condition of some of the patients and with the development of penicillin resistance for other patients, IV ceftriaxone was the drug of choice. Moreover, some of the patients refused to come three or four times a day for IV antibiotics or even to be admitted to the ENT ward; therefore, a single dose of IV ceftriaxone was more feasible for them, keeping in mind that overuse of such antibiotics should be avoided.

Furthermore, a rise in antibiotic resistance encourages physicians to choose broad-spectrum antibiotics, such as the third-generation cephalosporin (ceftriaxone), following the failure of amoxicillin and clavulanate, and second-generation cephalosporin, as effective treatment options.

A report from Japan found that third-generation cephalosporin was the most commonly prescribed antibiotic, followed by clarithromycin. ${ }^{11}$ Researchers at Kansai Medical University in Japan conducted a clinical and bacteriological study, administrating ceftriaxone once daily as a treatment for pediatric patients with upper respiratory tract infections. They found an efficacy rate of $95.6 \%,{ }^{12}$ whereas we found an efficacy rate of $89.7 \%$ when using the same drug to treat tonsillopharyngitis among all age groups.

Table 4 Reasons for initiating parenteral ceftriaxone

\begin{tabular}{lll}
\hline $\begin{array}{l}\text { Reasons of ceftriaxone } \\
\text { therapy }\end{array}$ & $\begin{array}{l}\text { Number of } \\
\text { patients }\end{array}$ & \% of patients \\
\hline $\begin{array}{l}\text { Failure of oral therapy } \\
\text { Inability to swallow }\end{array}$ & 36 & 37.1 \\
$\begin{array}{l}\text { Failure of oral therapy and } \\
\text { inability to swallow }\end{array}$ & 24 & 24.7 \\
Not documented & 14 & 21.6 \\
Referred from private sector & 2 & 14.4 \\
Total & 97 & 2.1 \\
\hline
\end{tabular}

Note: Parenteral ceftriaxone was initiated due to a variety of reasons.
A meta-analysis study comparing the effectiveness of cephalosporin relative to that of penicillin for the treatment of group A beta-hemolytic streptococcal pharyngitis showed that a short-duration treatment with cephalosporin provided a slightly better clinical outcome, a finding that ultimately supports our excellent outcome using this drug. ${ }^{12}$

A cross-sectional study was carried out among ENT patients with acute tonsillitis in the United Arab Emirates, to determine the antimicrobial prescription pattern. This study found that the majority of patients with acute tonsillitis were in the age group of 20-29 years, with higher male predominance, and the lowest number were in the geriatric age group. ${ }^{8}$ Our study similarly found the majority of OPAT patients were in the age group of 15-45 years, also having higher male predominance. The age group of 15-45 years suggests pharyngitis incidence rates increase among the adult population, specifically for parents of school-age children and for those whose occupation brings them into close contact with children. On the other hand, the lowest rates were found in the pediatric age group; however, this could be explained by the limited number of pediatric patients ( $\geq 2$ years of age) treated in the OPAT clinic. As such, a new pediatric OPAT clinic has recently opened in the BDF-RMS.

Culture and sensitivity tests of the throat swab were not carried out in $67 \%$ of the patients for a variety of reasons, including: they were already on oral antibiotics; they had been empirically treated following the Centers for Disease

Table 5 Antibiotic use before the use of ceftriaxone

\begin{tabular}{lll}
\hline Type of antibiotic & Number of patients & \% of patients \\
\hline Augmentin & 28 & 51.86 \\
Amoxicillin & 7 & 12.96 \\
Cefuroxime & 5 & 9.26 \\
Combined antibiotic & 9 & 16.66 \\
Others & 5 & 9.26 \\
Total & 54 & 100 \\
\hline
\end{tabular}

Notes: Oral antibiotics were used in 54 patients prior to the use of ceftriaxone. Presented are the various antibiotics that failed before ceftriaxone was initiated. 
Table 6 Types of organisms isolated from patients

\begin{tabular}{lll}
\hline Organisms & Number of patients & \% of patients \\
\hline Group A beta-hemolytic & 5 & 15.6 \\
Streptococcus & & \\
Staphylococcus & 5 & 15.6 \\
MRSA & 1 & 3.1 \\
No organism detected & 21 & 65.7 \\
Total & 32 & 100 \\
\hline
\end{tabular}

Note: Throat swab cultures isolated different organisms in 32 patients.

Abbreviation: MRSA, methicillin-resistant Staphylococcus aureus.

Control and Prevention (CDC) treatment guidelines; or they had a Centor criteria score that limited antibiotic therapy and diagnostic tests (for patients with 3 or 4 criteria). ${ }^{13}$ Group A beta-hemolytic Streptococci sp. (15.6\%) and Staphylococcus aureus $(15.6 \%)$ were the most commonly isolated bacterial organisms from the throat cultures. One case was found to have an MRSA-positive culture. This patient had been treated in another hospital before initiating his treatment at our hospital. It is possible that this patient had contracted the infection from the previous institution, and he was labeled as a case with unknown source of infection.

A review of the OPAT clinic at Auckland Hospital in New Zealand reported an $88 \%$ cure rate, while another study has found cure rates of over $90 \%$ for the treatment of a wide range of infections. ${ }^{8,9}$ Our OPAT cure rate was relatively similar, at $89 \%$, for treating acute tonsillopharyngitis. On the other hand, a study conducted at Regina Margherita Children Hospital in Italy reported the short-term therapy of streptococcal pharyngitis with ceftriaxone in 60 children. Patients were divided into randomized groups and treated with a single dose of ceftriaxone or multiple doses of ceftriaxone for 3 consecutive days. These researchers found that a clinical cure was obtained in $100 \%$ of the patients. ${ }^{8}$

Short bouts of both fever and throat soreness, recovery within 3 days of treatment, and fewer return visits explain the efficacy and excellent outcome of ceftriaxone therapy in the treatment of acute tonsillopharyngitis in our OPAT clinic. These findings support the results found at George Washington University Hospital, which indicated that ceftriaxone is an effective agent in the treatment of a variety of bacterial infections. ${ }^{14}$

A descriptive analysis of prospectively acquired data on the use of ceftriaxone in more than 1,300 OPAT visits over a 10 -year period in a UK center was conducted. This study found ceftriaxone had an excellent safety profile in the OPAT setting, and its broad spectrum of activity made it an established cure agent for a wide range of infections. ${ }^{10}$

Many studies have shown antimicrobial treatment in OPAT clinics results in lower costs when compared with the same treatment in the inpatient setting, which supports our findings. ${ }^{15}$ For example, cost effectiveness was calculated in 334 cases treated in the Sheffield OPAT clinic through the comparison

Table 7 OPAT Short-Form Patient's Satisfaction Questionnaire

\begin{tabular}{|c|c|c|c|c|c|c|}
\hline $\begin{array}{l}\text { Question } \\
\text { number }\end{array}$ & Questionnaire & $\begin{array}{l}\text { Strongly } \\
\text { disagree }\end{array}$ & Disagree & Uncertain & Agree & $\begin{array}{l}\text { Strongly } \\
\text { agree }\end{array}$ \\
\hline I & I am very satisfied with the medical care I received & I & 2 & 3 & 4 & 5 \\
\hline 2 & Doctors are good about explaining the reason for medical tests & & & & & \\
\hline 3 & I'm usually not kept waiting for a long time when I'm at OPAT & & & & & \\
\hline 4 & I think OPAT clinic has everything needed to provide complete medical care & & & & & \\
\hline 5 & The medical care I have been receiving is just about perfect & & & & & \\
\hline 6 & $\begin{array}{l}\text { During my medical visits, I'm always allowed to say everything that I think } \\
\text { it's important }\end{array}$ & & & & & \\
\hline 7 & $\begin{array}{l}\text { When I go for medical care, they are careful to check everything when } \\
\text { treating and examining me }\end{array}$ & & & & & \\
\hline 8 & The clinic hours when I can get medical care are convenient (good) for me & & & & & \\
\hline 9 & The medical staff that treats me known about the latest medical development & & & & & \\
\hline 10 & Doctors never expose me to unnecessary risks & & & & & \\
\hline II & My doctors treats me friendly and courteous & & & & & \\
\hline 12 & $\begin{array}{l}\text { Those who provide me my medical care sometimes not hurry too much } \\
\text { when they treat me }\end{array}$ & & & & & \\
\hline 13 & Places where I can get medical care are very conveniently located & & & & & \\
\hline 14 & If I have a medical question, I can reach a doctor for help without any problem & & & & & \\
\hline 15 & Doctors listen carefully to what I have to say & & & & & \\
\hline 16 & Doctors always do their best to keep me from worrying & & & & & \\
\hline 17 & I don't find it hard to get an appointment for medical care right way & & & & & \\
\hline 18 & My doctors are very competent and well-trained & & & & & \\
\hline
\end{tabular}

Note: This short-form questionnaire was used to assess patient satisfaction with OPAT clinic services.

Abbreviation: OPAT, outpatient parenteral antimicrobial therapy. 
of real costs of OPAT with estimated inpatient costs. It was found that treatment at the OPAT clinic cost only $41 \%$ of the equivalent inpatient treatment cost, ${ }^{15}$ a similar rate to our cost, which was $40 \%$ of the equivalent inpatient treatment cost.

Our hospital showed a $45.5 \%$ drop in admission rate for acute tonsillopharyngitis after starting the OPAT service clinic. This benefits both the hospital and the patients, as inpatient bed days are saved when patients are not admitted for parenteral antibiotics. A study conducted of Sheffield OPAT services between January 2006 and January 2008 found that the total number of bed-days saved through OPAT activity for different types of infections over a 2-year period was $4,034 .{ }^{15}$ Our study in the BDF-RMS found 301 bed-days were saved through OPAT services treatment of acute tonsillopharyngitis. These extra bed-days saved may be utilized for new admissions, and as well, may ultimately decrease both admission rates for acute tonsillopharyngitis and costs of care significantly.

\section{Conclusion}

In summary, this paper argued that the services provided through our OPAT clinic, using IV ceftriaxone for the management of acute tonsillopharyngitis, are safe, clinically effective (with low rates of complications/readmissions), and cost effective, and as well, render high levels of patient satisfaction.

\section{Acknowledgments}

The authors wish to thank Major General (Professor) Khalid Bin Ali Al Khalifa, Director of Royal Medical Services, for his support in launching the OPAT service clinic in the Bahrain Defense Force hospital.

\section{Disclosure}

The authors report no conflicts of interest in this work.

\section{References}

1. Rucker RW, Harrison GM. Outpatient intravenous medications in the management of cystic fibrosis. Pediatrics. 1974;54(3):358-360.

2. Chapman, ALN. Outpatient parenteral antimicrobial therapy. BMJ. 2013;346:27-30.

3. Al Ansari A, Al Alawi S, Al Qahtani M, Darwish A. Outpatient parenteral antimicrobial therapy (OPAT) in the Kingdom of Bahrain: Efficacy, patient satisfaction and cost effectiveness. Open Infect Dis J. 2013;7:90-95.

4. Gouliouris T, Bioh G, Karas JA. Outpatient parenteral antimicrobial therapy (OPAT) as a nurse-led service: A district general hospital perspective. Poster presented at: Program and abstracts of the joint conference of the Imperial College London and British Society for Antimicrobial Chemotherapy; December 5; 2011; London.

5. Pichichero ME. Pathogen shifts and changing cure rates for otitis media and tonsillopharyngitis. Clin Pediatr (Phila). 2006;45(6):493-502.

6. Pavesio D, Pecco P, Peisino MG. Short-term treatment of streptococcal tonsillitis with ceftriaxone. Chemotherapy. 1988;34 Suppl 1: S34-S38.

7. Bisno AL. Acute pharyngitis. N Engl J Med. 2001;344(3):205-211.

8. John LJ, Cherian M, Sreedharan J, Cherian T. Patterns of antimicrobial therapy in acute tonsillitis: A cross-sectional hospital-based study from UAE. An Acad Bras Cienc. 2014;86(1):451-457.

9. Upton A, Ellis-Pegler RB, Woodhouse A. Outpatient parenteral antimicrobial therapy (OPAT): A review of experience at Auckland Hospital. N Z Med J. 2004;117(1200):U1020.

10. Duncan CJ, Barr DA, Seaton RA. Outpatient parenteral antimicrobial therapy with ceftriaxone, a review. Int J Clin Pharm. 2012;34(3): 410-417.

11. Higashi T, Fukuhara S. Antibiotic prescriptions for upper respiratory tract infection in Japan. Intern Med. 2009;48(16):1369-1375.

12. Hasui M, Kobayashi Y, Harada Y, et al. [Clinical and bacteriological studies of ceftriaxone (CTRX) once daily administration in pediatric patients with respiratory tract infections]. Jpn J Antibiot. 2001;54(10): 532-540. Japanese.

13. Cooper RJ, Hoffman JR, Bartlett JG, et al; American Academy of Family Physicians; American College of Physicians-American Society of Internal Medicine; Centers for Disease Control. Principles of appropriate antibiotic use for acute pharyngitis in adults: background. Ann Intern Med. 2001;134(6):509-517.

14. Epstein JS, Hasselquist SM, Simon GL. Efficacy of ceftriaxone in serious bacterial infections. Antimicrob Agents Chemother. 1982;21(3): 402-406.

15. Chapman AL, Dixon S, Andrews D, Lillie PJ, Bazaz R, Patchett JD. Clinical efficacy and cost-effectiveness of outpatient parenteral antibiotic therapy (OPAT): a UK perspective. J Antimicrob Chemother. 2009;64(6):1316-1324.

\section{Publish your work in this journal}

Infection and Drug Resistance is an international, peer-reviewed openaccess journal that focuses on the optimal treatment of infection (bacterial, fungal and viral) and the development and institution of preventive strategies to minimize the development and spread of resistance. The journal is specifically concerned with the epidemiology of antibiotic

\section{Dovepress}

resistance and the mechanisms of resistance development and diffusion in both hospitals and the community. The manuscript management system is completely online and includes a very quick and fair peerreview system, which is all easy to use. Visit http://www.dovepress.com/ testimonials.php to read real quotes from published authors. 